\title{
Male Adenoid Cystic Carcinoma of the Breast
}

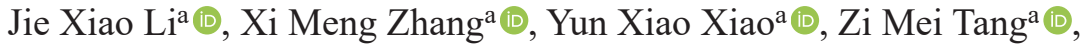 \\ Tao Huanga, b (i), Jie Minga, b (B)
}

\begin{abstract}
Adenoid cystic carcinoma (ACC) of the breast, a rare malignancy that makes up less than $0.1 \%$ of all breast malignancies, is much rarer in males than in females. Due to the rarity of this disease, an optimal treatment strategy for male breast ACC has not been established, and therapy for male patients is currently based on guidelines for female patients. According to previous reported cases, some authors believe that male breast ACC may have higher invasive potential than female breast ACC and the prognoses in male patients may be worse than those in female patients. Therefore, a more proactive diagnosis and treatment regimen may be required. However, the clinical feature of our case is inconsistent with this view. Herein we report the case of a 24 -year-old male without any antecedent medical or family history who presented with a slow-growing lump on his left chest wall for 5 years. The patient initially underwent lumpectomy, and the mass was pathologically diagnosed as breast ACC. Systemic examination was performed, and no evidence of distant metastasis was found. Then, he received modified radical mastectomy and ipsilateral axillary lymph node dissection. The mastectomy pathological examination revealed that no cancerous tissue was detected around the primary tumor bed, and all 22 axillary lymph nodes were negative. The patient did not receive postoperative chemotherapy, radiotherapy or endocrine therapy and remained well after 28 months of follow-up. In this study, we review the literature and summarize the clinical manifestations, imaging and histopathological characteristics, treatments and outcomes of male breast ACC. We share our experience in the hopes that this evidence will aid in the development of better therapeutics.
\end{abstract}

Keywords: Adenoid cystic carcinoma; Breast; Male; Treatment; Prognosis; Case report

\section{Introduction}

Adenoid cystic carcinoma (ACC) was first discovered by Rob-

Manuscript submitted September 12, 2021, accepted October 13, 2021

Published online December 2, 2021

aDepartment of Breast and Thyroid Surgery, Union Hospital Affiliated to Tongji Medical College, Huazhong University of Science and Technology, 1277 Jiefang Avenue, Wuhan 430022, China

${ }^{\mathrm{b}}$ Corresponding Author: Jie Ming and Tao Huang, Department of Breast and Thyroid Surgery, Union Hospital Affiliated to Tongji Medical College, Huazhong University of Science and Technology, 1277 Jiefang Avenue, Wuhan 430022, China. Email: mingjiewh@126.com and huangtaowh@163.com

doi: https://doi.org/10.14740/jmc3790 in and Laboulbene in 1853 and described as "tumeur heteradenique" [1]. Then ACC was first used in breast oncology by Geschikter in 1945 [2]. ACC is commonly seen in the major and minor salivary glands, though cases in the breast, skin, lung and bronchus, lacrimal gland, cervix, and various other sites have also been reported [3]. Breast ACC is a very rare primary breast cancer, making up less than $0.1 \%$ of all breast malignancies [4]. Of these, male patients are even rarer. Male breast ACC was first reported in 1969, and a total of 16 cases have been recorded [5-20]. Breast ACC is a type of basal-like breast cancer that is generally negative for estrogen receptor (ER), progesterone receptor (PR), and human epidermal growth factor receptor 2 (HER-2). Unlike other triple-negative breast cancers, however, breast ACC has a favorable prognosis. Clinical manifestations and imaging analyses of breast ACC are nonspecific; thus, histopathological examination is necessary for accurate diagnosis [4]. However, diagnostic and therapeutic recommendations for male patients have yet to be determined due to the scarcity of existing cases. Currently, the diagnostic and therapeutic modalities for male breast ACC rely on guidelines developed in female patients. Therefore, we hope that this report describing a rare case of a 24-year-old male patient with breast ACC will provide evidence needed for the development of diagnostic and treatment standards among this group of patients.

\section{Case Report}

\section{Investigations}

A 24-year-old man had a palpable tough mass on his left chest wall accompanied by mild pain and no redness of the skin. The lump developed in 2014 and had continued to grow slowly during the 5 years of follow-up with no medical intervention. The patient had no previous medical history. Physical examination found a hard, regularly shaped, mobile mass of approximately $1 \mathrm{~cm}$ in diameter which was palpable under the left areola. Lymph nodes of axilla were not palpable.

\section{Diagnosis}

A March 2017 breast ultrasound revealed a $0.8 \times 0.5 \mathrm{~cm}$ hypoechoic mass on the retroareolar region of the left breast (Fig. 1a) (The earliest ultrasound results in 2014 were una- 


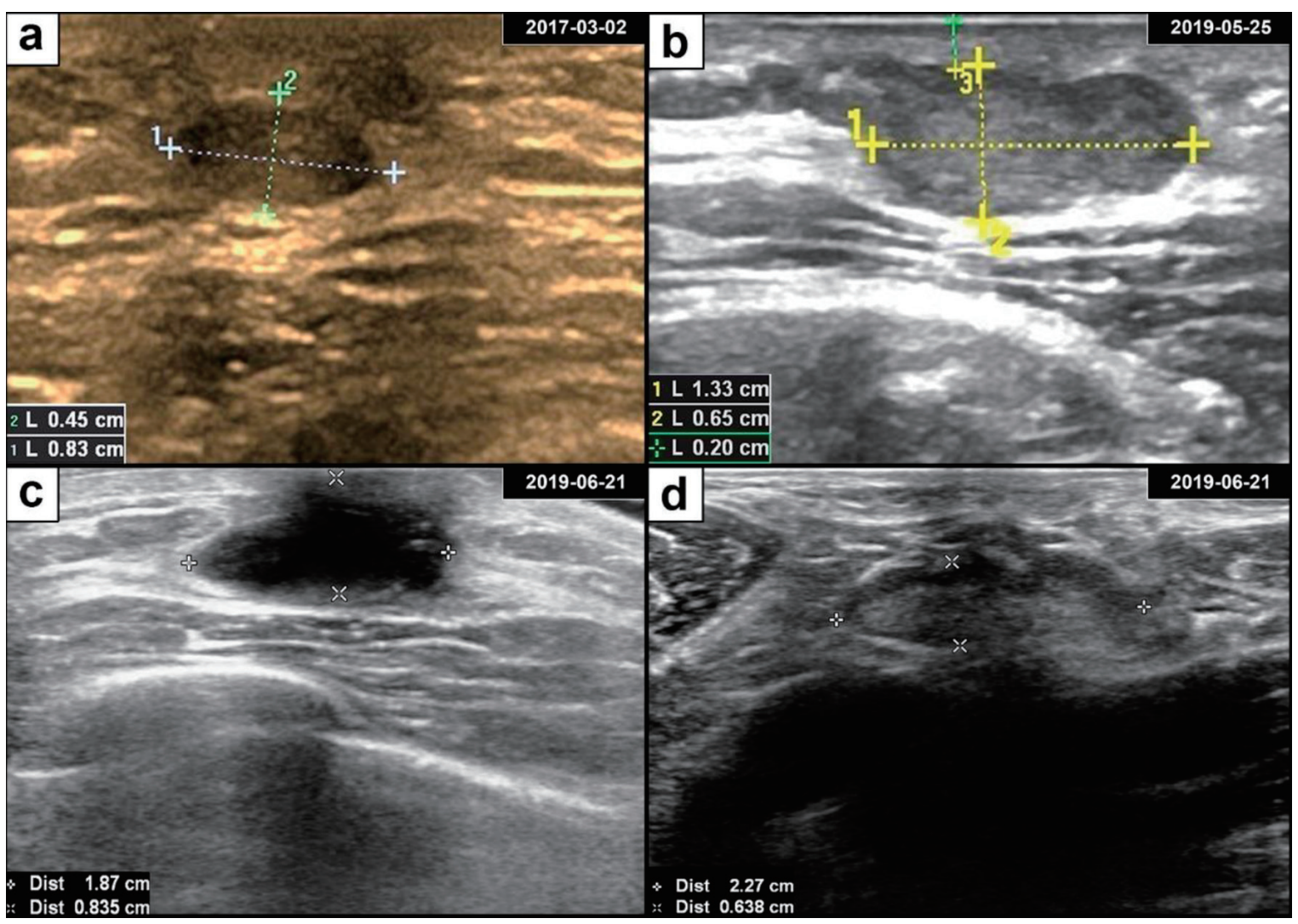

Figure 1. Breast adenoid cystic carcinoma ultrasound and postoperative findings. (a) Ultrasonography revealed a well-defined oval hypoechoic mass in the retroareolar region of the left breast. (b) The subareolar mass progressively enlarged over the course of 2 years. (c) Ultrasonography showed a hypoechoic area after lumpectomy. (d) Ultrasonography of an enlarged axillary lymph node with a clear boundary.

vailable). In May 2019, the mass had grown to $1.3 \times 0.7 \mathrm{~cm}$ in size and was hypoechoic with a clear margin and uneven internal echo (Fig. 1b). Therefore, the patient underwent lumpectomy in May 2019. According to the pathological examination results, the mass was described as a biphasic tumor composed of glandular epithelial cells and myoepithelial cells arranged in a tubular, cribriform, solid structure, which supports the diagnosis of breast ACC (Fig. 2). Immunohistochemical staining showed that the tumor cells were negative for ER, PR and HER-2 and positive for E-cadherin, P120-catenin, P63, CK5/6, and CD117. Approximately 20\% of the cells expressed Ki67. Then the patient immediately returned to the Department of Breast and Thyroid Surgery for further treatment and received a breast ultrasound and ${ }^{18} \mathrm{~F}$-fluorodeoxyglucose positron emission tomography/computed tomography ( ${ }^{18} \mathrm{~F}-\mathrm{FDG}$ PET-CT). The breast ultrasound revealed a $1.8 \times 0.9 \mathrm{~cm}$ hypoechoic area under the areola of the left breast (possible postoperative change) (Fig. 1c). Lymph nodes were visible in the bilateral axilla and left clavicle, and an enlarged lymph node approximately $2.3 \times 0.6 \mathrm{~cm}$ in size was observed in the left axilla (Fig. 1d). The ${ }^{18} \mathrm{~F}-\mathrm{FDG}$ PET-CT revealed a disordered subcutaneous structure in the left breast region accompanied by increased metabolism, indicating postoperative changes. Small lymph nodes with low metabolism were found in the bilateral neck and axilla. No significant metabolic abnormalities were observed anywhere else in the patient (Fig. 3).

\section{Treatment}

Based on the breast ultrasound, ${ }^{18}$ F-FDG PET-CT, pathological examination and the preference of the patient, we performed modified radical mastectomy and left axillary lymph node dissection in June 2019. After the surgery, the patient did not receive subsequent radiotherapy, chemotherapy or endocrine therapy.

\section{Follow-up and outcomes}

Mastectomy histopathological examination showed no residual carcinoma tissue around the primary tumor bed in the left breast. No vascular tumor embolus was evident by pathological analysis. No nerve invasion was detected, and the surgical margin was negative. None of the 22 ipsilateral axillary lymph nodes resected showed metastatic carcinoma. The patient recovered well and was discharged 1 week later. The patient remained healthy over 28 months of regular follow-up, with no signs of local relapse or distant metastases (Supplementary 


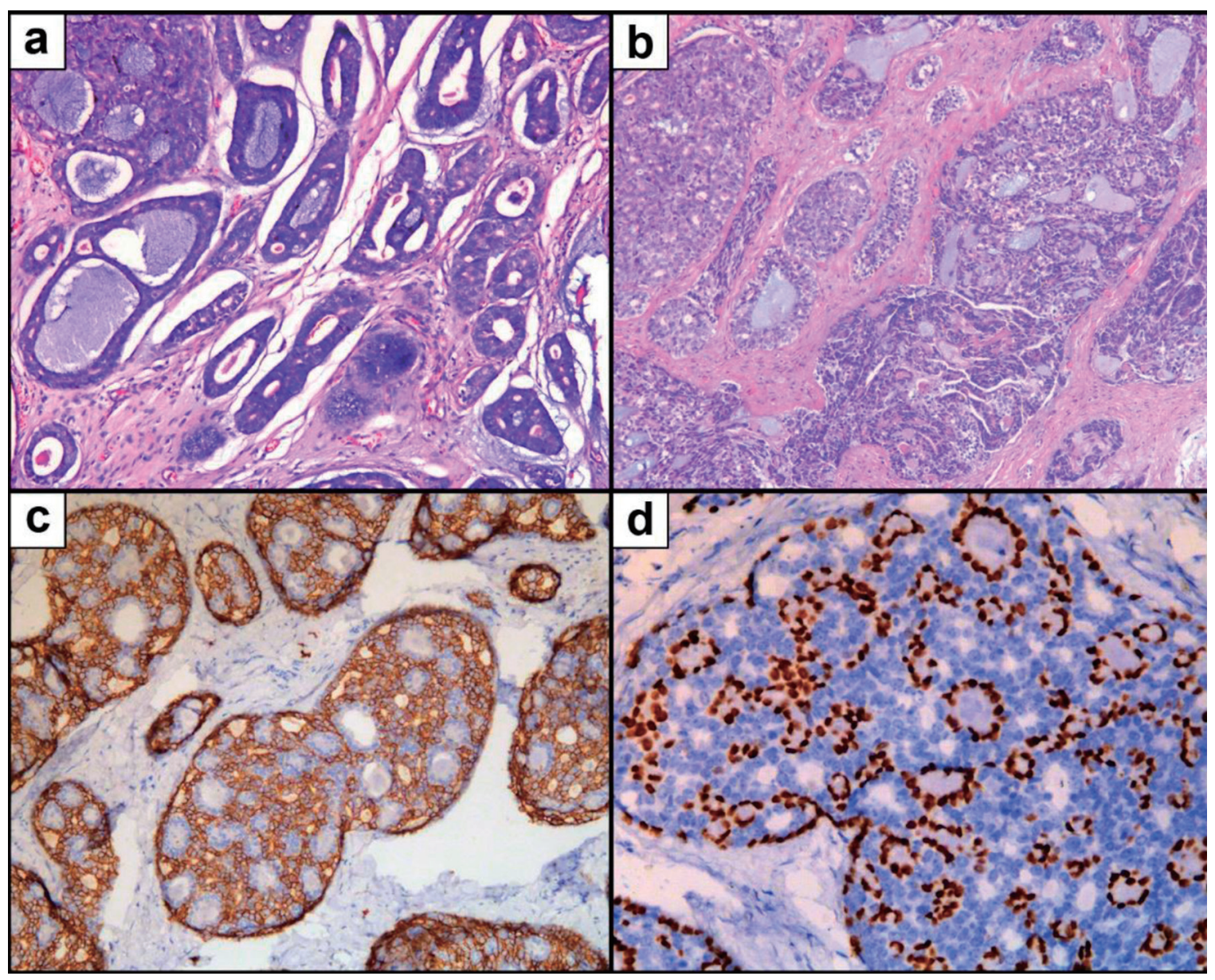

Figure 2. Microscopic pathology of breast adenoid cystic carcinoma. (a) H\&E staining showing an cribriform growth pattern. (b) H\&E staining showing tubular and solid growth patterns. (c) IHC staining for CD117. (d) IHC staining for P63. H\&E: hematoxylin and eosin stain; IHC: immunohistochemistry.

Material 1, www.journalmc.org).

\section{Discussion}

The incidence of breast ACC is very low, comprising less than $0.1 \%$ of all breast carcinomas [4]. Therefore, male breast ACC is very rare; indeed, there have been only 17 reported cases since 1969 [5-20]. Of these, nine cases were reported within the past decade, which may indicate a trend toward increased incidence [13-20]. The susceptibility of various racial subpopulations to breast ACC is currently unknown, though a previous report indicated that black females have a lower incidence rate than white females [21]. Interestingly, eight out of 12 male cases reported in the past 30 years were reported in Asia [12$17,19]$. The median age of breast cancer onset is somewhat higher in males (about 67 years) than females (about 62 years) [22]. The incidence of breast cancer is positively correlated with age in both males and females [22]. The age of onset for male breast ACC is lower (median 41.5 years) than that seen in male breast cancer patients (Table 1 [5-20]). For female breast ACC patients, the median age of onset is $58-66$ years old [21,
23-26]. The case we report here is from a 24-year-old male.

The dominant manifestation of breast ACC in males is a hard palpable subareolar mass that may be accompanied by pain or tenderness. Other symptoms include skin ulcers and nipple retraction $[11,12,17]$. Nipple discharge has not been reported in any of the available cases. Distant metastases have also been reported as an initial symptom $[15,20]$. In female breast ACC, masses are usually detected shortly before seeking medical care [27]. However, due to insufficient awareness of male breast diseases, male patients typically report that their mass has been present for several years by the time of diagnosis [27]. Breast ACC usually appears to be unifocal but can occasionally be multifocal. There is no significant difference in incidence between the left and right breasts [21]. For male patients, no bifocal or multifocal cases have been reported. Tumor size is generally larger in male breast cancer patients than in female patients [28], which is consistent with the data collected from male and female breast ACC patients. The mean tumor diameter in female breast ACC patients is $1.8-2.2 \mathrm{~cm}$ $[3,21,29]$ and the median is $1.8-2.2 \mathrm{~cm}[21,23]$. In male breast ACC patients, the reported mean tumor size is 1.2 - 5 $\mathrm{cm}$ and the median is $2.6 \mathrm{~cm}$ (Table 1). The tumor in this case 

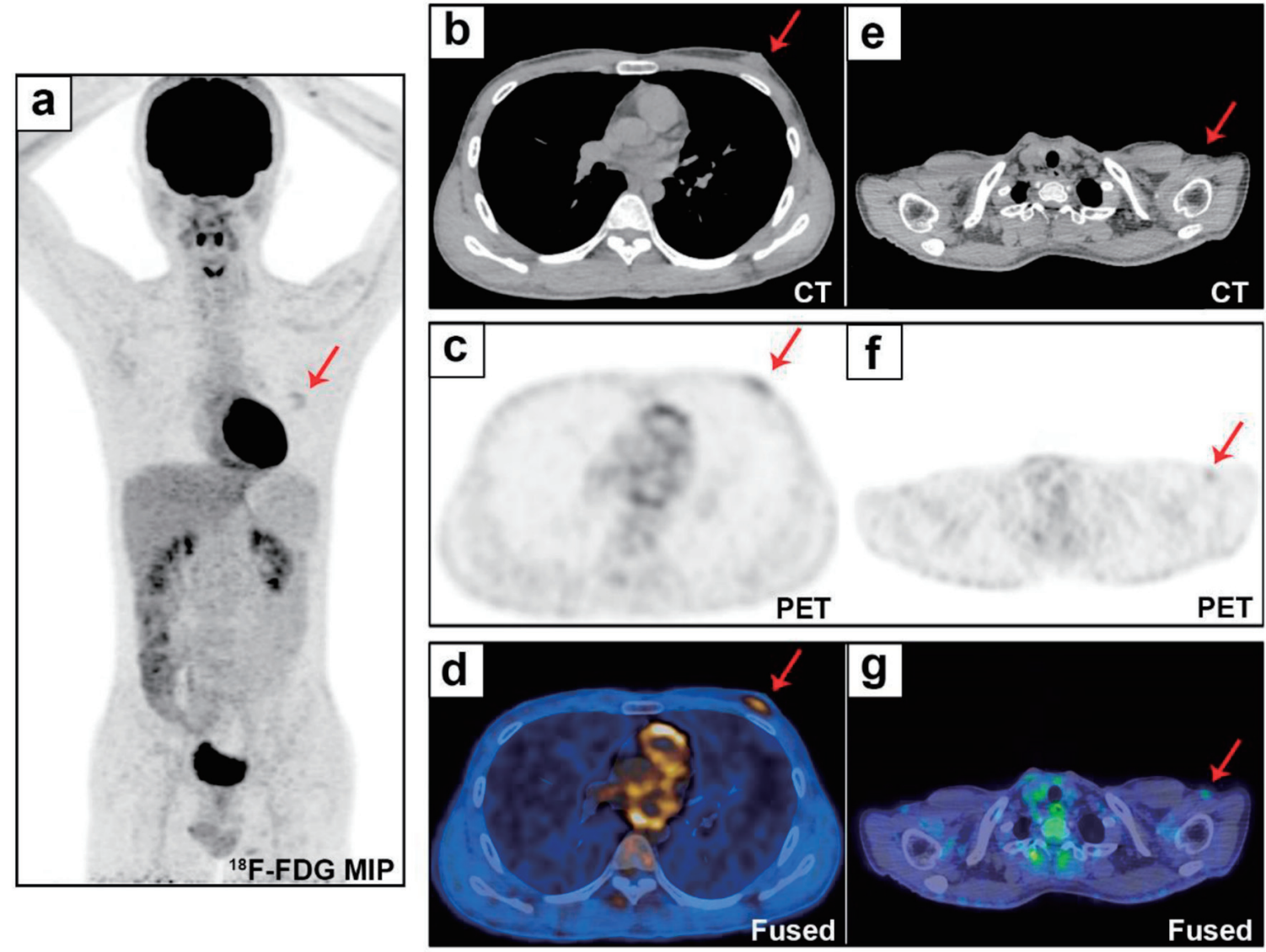

Figure 3. Whole-body evaluation by ${ }^{18}$ F-FDG PET/CT. The ${ }^{18} \mathrm{~F}-\mathrm{FDG}$ PET/CT MIP (a) shows uptake (SUVmax 2.5) in the left breast lesion ( $b$ and d, red arrows), with a disordered tissue structure (c, red arrow). Small lymph nodes were found in the bilateral neck and bilateral axillary regions (e-g, red arrows, SUVmax 1.65). ${ }^{18} \mathrm{~F}-\mathrm{FDG}$ PET/CT: ${ }^{18} \mathrm{~F}$-fluorodeoxyglucose positron emission tomography/computed tomography; MIP: maximum intensity projection; SUVmax: maximum standardized uptake value.

measured only $1.3 \mathrm{~cm}$, which is smaller than the average.

Due to the rarity of the disease, the imaging features of male breast ACC have not been well described. Compared with female breast ACC patients, male cases have very similar nonspecific ultrasonic characteristics, including clear or indistinct boundaries, irregular shapes, and hypoechoic and heterogeneous masses $[15,16,19,30]$. On mammography, female breast ACC often manifests as an irregular, lobulated, heterogeneous mass with indistinct or microlobulated edges [30]. However, the dominant features in males are spiculated shadows in the subareolar area [16]. Thus far, magnetic resonance imaging (MRI) features of male breast ACC have only been mentioned by Pang et al and include oval nodules, spiculated edges, T1WI hypointensity, and T2WI hyperintensity. Dynamic contrast-enhanced MRI time intensity curves have revealed that tumor enhancement is fast and is followed by a plateau [19]. In female breast ACC cases, the common features include $\mathrm{T} 2$ hyperintense in large foci and $\mathrm{T} 2$ equal intense in small foci [31,32]. However, the tumor in this case was $1.2 \mathrm{~cm}$ in diameter with T2WI hyperintensity, which is quite different from the characteristics seen in female cases [19].

Unique histopathological features are the major evidence used to diagnose breast ACC. Breast ACC is a kind of biphasic tumor composed of basaloid and luminal epithelial cells. The histological features are similar to those of salivary gland ACC. The tumor cells are distributed around small cysts, forming both true glands and pseudoglandular spaces containing eosinophilic basement membrane material and basophilic mucin. The cells may show cribriform, tubular, or solid growth patterns, either alone or in combination $[4,27,32]$. However, unlike salivary gland ACC, breast ACC rarely features nerve infiltration [33]. Ro et al first classified breast ACC into three grades based on the ratio of solid components within the lesion. Grade I indicates no solid growth. In grade II tumors, the proportion of solid growth is less than $30 \%$, while in grade III tumors, the proportion is more than $30 \%$ [34]. Ro et al also proposed that grade II and III tumors are larger in size and are more prone to relapse [34], though this remains controversial [4]. Breast ACC has similar immunohistochemical characteristics to basal-like breast cancer and is often negative for ER, PR and HER-2 [29]. However, other studies have reported ER and PR expression in 0-46\% and $0-36 \%$ of breast ACC patients, respectively [21, 23, 24, 29, $35]$. All of the reported male cases were negative for PR, though two cases were weakly positive for ER [12,16]. In the dual-cell population of breast ACC, glandular epithelial cells express cytokeratin (CK)7, CK8/18, epithelial membrane antigen (EMA) 
Table 1. Summary of Male Adenoid Cystic Carcinoma of Breast

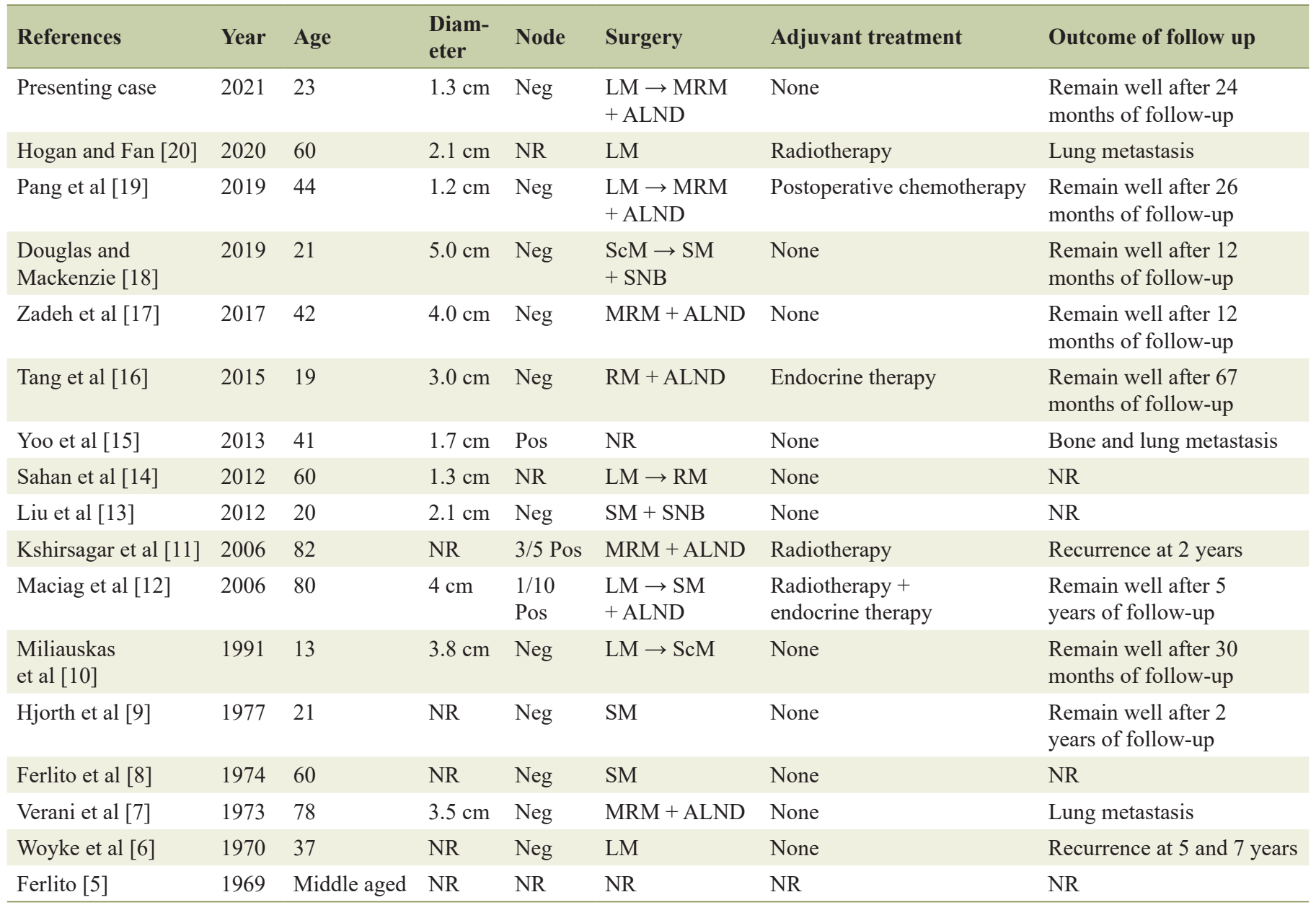

Neg: negative; Pos: positive; NR: no report; LM: lumpectomy; MRM: modified radical mastectomy; ALND: axillary lymph node dissection; ScM: subcutaneous mastectomy; SM: simple mastectomy; SNB: sentinel node biopsy; RM: radical mastectomy.

and CD117 (C-kit). Fodrin, E-cadherin and $\beta$-catenin are usually expressed. Myoepithelial cells are immunoreactive for CK5/6, CK14, CK17, P63, smooth muscle actin (SMA), calponin, and vimentin $[4,27]$. The Ki67 index ranges from $4 \%$ to $70 \%$, and is higher in tumors with solid components (grade II-III) than in tumors without solid components (grade I) [27]. In our case, the tumor would be histologically classified as grade II with a Ki67 index of $20 \%$, a typical ER/PR/HER-2 triple-negative genotype and a biphasic pattern of epithelial cells (CD117 positive) and myoepithelial cells ( $\mathrm{P} 63, \mathrm{CK} 5 / 6$ positive).

Unlike basal-like/triple-negative breast cancer or salivary gland ACC, which are aggressive, female breast ACC usually has more favorable biological characteristics and prognosis. Previous studies have reported that the axillary lymph node metastasis rate is $0-5.1 \%[3,21,23-25,29]$, the local recurrence rate is $0-13 \%[23,33]$, the distant metastasis rate is $0.3-$ $13 \%[3,21,23,24,33]$, and the 10 -year survival rate is $86-94 \%$ $[3,21,23,25,33,35]$. To date, the prognosis of male breast ACC is still obscure. Among the reported cases of male breast ACC, three (21.4\%) had lymph node metastasis, two (15.4\%) had local recurrence, and three $(23 \%)$ had distant metastasis.
The majority of the eight cases with no relapse had a followup period significantly shorter than the median follow-up time of female patients (59 - 84 months) $[23,24,33,35]$. Moreover, Millar et al mentioned in their retrospective study that a 53-year-old male ACC patient died from lung, bone and abdominal metastases 7 months after receiving a simple mastectomy and adjuvant radiotherapy [26]. Therefore, some authors consider that male breast ACC may be more aggressive and have poorer prognosis than female $[16,17,19]$. Nevertheless, there are insufficient data to support this speculation.

Surgery is the main treatment option for breast ACC [31]. Most female patients receive breast-conserving surgery. Since axillary lymph node metastasis is rare, routine axillary lymph node dissection is generally considered unnecessary for female breast ACC [23-25, 29]. For the use of adjuvant radiotherapy, there is no universal standard for the use of adjuvant radiotherapy. However, several studies have reported positive surgical margins after breast-conserving therapy [28]. Moreover, other studies have demonstrated that adjuvant radiotherapy is effective at improving overall and disease-specific survival rates in female patients $[35,36]$. Therefore, postoperative ad- 
juvant radiotherapy is recommended in most studies [35-37]. Chemotherapy and endocrine therapy are infrequently used for the treatment of breast ACC. Only $11 \%$ and $8 \%$ of female patients received adjuvant chemotherapy and endocrine therapy, respectively [29]. Except for the cases reported by Woyke et al and Hogan et al in 1970 and 2020 [6, 20], most (13 of 15) male patients underwent simple mastectomy or modified radical mastectomy [7-14, 16-19]. Ipsilateral axillary lymph node dissection was performed in seven out of the 15 men [7, 11, 12, 16, $17,19]$, and sentinel lymph node biopsy was performed in two cases $[13,18]$. Three patients received adjuvant radiotherapy; in one case the radiotherapy was performed following wide resection of a locally recurrent lesion [11, 12, 20]. One patient underwent postoperative chemotherapy with cyclophosphamide, epirubicin, and docetaxel, and two patients with weak immunoreactivity for ER received endocrine therapy $[12,16$, 19]. In the case reported here, the patient initially underwent lumpectomy, and the tumor was pathologically diagnosed as breast ACC. After taking the potentially aggressive biological behavior of male breast ACC into consideration and respecting the patients' wishes, we performed modified radical mastectomy and axillary lymph node dissection. However, pathological examination revealed that there was no cancerous tissue around the primary tumor bed, all 22 axillary lymph nodes were negative, and there was no perineural or lymphovascular invasion. From the limited data mentioned above, although the axillary lymph node metastasis rate of breast ACC in males is slightly higher than that in females, the axillary lymph nodes in most reported male patients are still negative (Table 1). Therefore, axillary lymph node dissection may be unnecessary for male breast ACC and routine sentinel lymph node biopsy may be a better option. The patient did not undergo adjuvant radiotherapy or chemotherapy after surgery. In addition, a multiinstitutional study of breast ACC reported that the median time to locoregional recurrence and distant metastasis was 48 and 25.5 months, respectively. The longest time to local recurrence or distant relapse was 161 months [38]. Therefore, we recommended that our patient undergo regular follow-ups at $3-6$ month intervals for the first 2 years and then every year for at least 10 years. The patient remained in good condition with no recurrence during a 28 -month follow-up period.

In conclusion, breast ACC is a rare malignant neoplasm with an excellent prognosis. Its epidemiological characteristics, clinical manifestations, imaging characteristics, biological features, prognosis and treatment regimens may vary between sexes. According to previous reports, the prognosis of male breast ACC may be worse than female patients due to potentially aggressive behavior and the neglect of the disease. However, whether to choose a more radical treatment strategy for male breast ACC than female patient remains to be determined due to lack of clinical data. More long-term follow-up studies are necessary to determine the optimal treatment protocol of male breast ACC.

\section{Supplementary Material}

Suppl 1. Timeline of the male breast ACC case. ACC: adenoid cystic carcinoma.

\section{Acknowledgments}

None to declare.

\section{Financial Disclosure}

This report received no specific grant from any funding agency in the public, commercial, or not-for-profit sectors.

\section{Conflict of Interest}

The authors have no conflict of interest to disclose.

\section{Informed Consent}

Written informed consent for publication of his clinical details and/or clinical images was obtained from the patient.

\section{Author Contributions}

Li JX reviewed the literature and drafted the manuscript; Huang $\mathrm{T}$ and Ming $\mathrm{J}$ conceived and designed this case report and revised the manuscript; Zhang XM was the patient's attending surgeon and helped draft the paper; Xiao YX and Tang ZM contributed to the literature review and manuscript drafting; all authors read and approved the final version of the submitted manuscript.

\section{Data Availability}

The authors declare that data supporting the findings of this study are available within the article.

\section{Abbreviations}

ACC: adenoid cystic carcinoma; ER: estrogen receptor; PR: progesterone receptor; HER-2: human epidermal growth factor receptor $2 ;{ }^{18} \mathrm{~F}-\mathrm{FDG}$ PET-CT: ${ }^{18} \mathrm{~F}$-fluorodeoxyglucose positron emission tomography/computed tomography; CK: cytokeratin; EMA: epithelial membrane antigen; SMA: smooth muscle actin

\section{References}

1. Cantu G. Adenoid cystic carcinoma. An indolent but aggressive tumour. Part A: from aetiopathogenesis to diagnosis. Acta Otorhinolaryngol Ital. 2021;41(3):206-214.

2. Geschickter CF, Copeland MM. Diseases of the breast: diagnosis, pathology, treatment. Philadelphia: J.B. Lippincott; 1945. 
3. Li N, Xu L, Zhao H, El-Naggar AK, Sturgis EM. A comparison of the demographics, clinical features, and survival of patients with adenoid cystic carcinoma of major and minor salivary glands versus less common sites within the Surveillance, Epidemiology, and End Results registry. Cancer. 2012;118(16):3945-3953.

4. Miyai K, Schwartz MR, Divatia MK, Anton RC, Park YW, Ayala AG, Ro JY. Adenoid cystic carcinoma of breast: Recent advances. World J Clin Cases. 2014;2(12):732741.

5. Ferlito A. [Adenoid cystic carcinoma of the male breast]. Riv Anat Patol Oncol. 1969;35:441-455.

6. Woyke S, Domagala W, Olszewski W. Fine structure of mammary adenoid cystic carcinoma. Pol Med J. 1970;9(5):1140-1148.

7. Verani RR, Van der Bel-Kahn J. Mammary adenoid cystic carcinoma with unusual features. Am J Clin Pathol. 1973;59(5):653-658.

8. Ferlito A, Di Bonito L. Adenoid cystic carcinoma of the male breast: report of a case. Am Surg. 1974;40(1):72-76.

9. Hjorth S, Magnusson PH, Blomquist P. Adenoid cystic carcinoma of the breast. Report of a case in a male and review of the literature. Acta Chir Scand. 1977;143(3):155158.

10. Miliauskas JR, Leong AS. Adenoid cystic carcinoma in a juvenile male breast. Pathology. 1991;23(4):298-301.

11. Kshirsagar AY, Wader JV, Langade YB, Jadhav KP, Zaware SU, Shekhar N. Adenoid cystic carcinoma of the male breast. Int Surg. 2006;91(4):234-236.

12. Maciag H, Ziolkowski P, Wrzecion S, Kolodziej P. Adenoid cystic carcinoma of the breast in an 80-year-old male - a case report. Wspolczesna Onkologia. 2006;10(7):340.

13. Liu J, Jia W, Zeng Y, Deng H, Rao N, Su F. Adolescent male adenoid cystic breast carcinoma. Am Surg. 2012;78(5):E288-289.

14. Sahan EK, Karinoglu U, Igdem AA, Erdogan N. Adenoid cystic carcinoma in male breast: a case report. Virchows Arch. 2012;461(Suppl 1):S244.

15. Yoo SJ, Lee DS, Oh HS, Kim HJ, Kim MH, Ahn YC, Kang GH, et al. Male breast adenoid cystic carcinoma. Case Rep Oncol. 2013;6(3):514-519.

16. Tang P, Yang S, Zhong X, Yao J, Zhang Y, Dong H, Li G. Breast adenoid cystic carcinoma in a 19-year-old man: a case report and review of the literature. World J Surg Oncol. 2015;13:19.

17. Yahya Zadeh H, Aminmozaffari S, Vand Rajabpour M. Male adenoid cystic carcinoma of breast. International Journal of Cancer Management. 2017;10(9):e7827.

18. Mackenzie J, Douglas C. Gynecomastia or rare malignancy? A young man with an unremarkable history proves to have adenoid cystic carcinoma of the breast. Breast J. 2020;26(3):502-504.

19. Pang W, Wang Z, Jin X, Zhang Q. Adenoid cystic carcinoma of the breast in a male: A case report. Medicine (Baltimore). 2019;98(32):e16760.

20. Hogan KO, Fan F. Diagnosis of metastatic adenoid cystic carcinoma of the breast on pleural fluid cytology in a 60-year-old male. Diagnostic Cytopathology. 2020;49(4):E172-E174.
21. Ghabach B, Anderson WF, Curtis RE, Huycke MM, Lavigne JA, Dores GM. Adenoid cystic carcinoma of the breast in the United States (1977 to 2006): a populationbased cohort study. Breast Cancer Res. 2010;12(4):R54.

22. Hassett MJ, Somerfield MR, Baker ER, Cardoso F, Kansal KJ, Kwait DC, Plichta JK, et al. Management of male breast cancer: ASCO guideline. J Clin Oncol. 2020;38(16):1849-1863.

23. Arpino G, Clark GM, Mohsin S, Bardou VJ, Elledge RM. Adenoid cystic carcinoma of the breast: molecular markers, treatment, and clinical outcome. Cancer. 2002;94(8):2119-2127.

24. Defaud-Henon F, Tunon-de-Lara C, Fournier M, Marty M, Velasco V, de Mascarel I, MacGrogan G. [Adenoid cystic carcinoma of the breast: clinical, histological and immunohistochemical characterization]. Ann Pathol. 2010;30(1):7-16.

25. Thompson K, Grabowski J, Saltzstein SL, Sadler GR, Blair SL. Adenoid cystic breast carcinoma: is axillary staging necessary in all cases? Results from the California Cancer Registry. Breast J. 2011;17(5):485-489.

26. Millar BA, Kerba M, Youngson B, Lockwood GA, Liu FF. The potential role of breast conservation surgery and adjuvant breast radiation for adenoid cystic carcinoma of the breast. Breast Cancer Res Treat. 2004;87(3):225-232.

27. Brogi E. Rosen's breast pathology. fourth edition ed. Hoda SA, Koerner FC, Brogi E, Rosen PP, editors. Philadelphia: Lippincott Williams \& Wilkins; 2014:725-748.

28. Giordano SH, Cohen DS, Buzdar AU, Perkins G, Hortobagyi GN. Breast carcinoma in men: a population-based study. Cancer. 2004;101(1):51-57.

29. Kulkarni N, Pezzi CM, Greif JM, Suzanne Klimberg V, Bailey L, Korourian S, Zuraek M. Rare breast cancer: 933 adenoid cystic carcinomas from the National Cancer Data Base. Ann Surg Oncol. 2013;20(7):2236-2241.

30. Tang W, Peng WJ, Gu YJ, Zhu H, Jiang TT, Li C. Imaging manifestation of adenoid cystic carcinoma of the breast. J Comput Assist Tomogr. 2015;39(4):523-530.

31. Treitl D, Radkani P, Rizer M, El Hussein S, Paramo JC, Mesko TW. Adenoid cystic carcinoma of the breast, 20 years of experience in a single center with review of literature. Breast Cancer. 2018;25(1):28-33.

32. Agafonoff S, Sobolewski A, Braverman TS. Adenoid cystic carcinoma of the breast - Discordant size on imaging and pathology: a case report and review of literature. Ann Med Surg (Lond). 2019;43:1-4.

33. Khanfir K, Kallel A, Villette S, Belkacemi Y, Vautravers C, Nguyen T, Miller R, et al. Management of adenoid cystic carcinoma of the breast: a rare cancer network study. Int J Radiat Oncol Biol Phys. 2012;82(5):21182124.

34. Ro JY, Silva EG, Gallager HS. Adenoid cystic carcinoma of the breast. Hum Pathol. 1987;18(12):1276-1281.

35. Sun JY, Wu SG, Chen SY, Li FY, Lin HX, Chen YX, He ZY. Adjuvant radiation therapy and survival for adenoid cystic carcinoma of the breast. Breast. 2017;31:214-218.

36. Coates JM, Martinez SR, Bold RJ, Chen SL. Adjuvant radiation therapy is associated with improved survival for adenoid cystic carcinoma of the breast. J Surg Oncol. 
2010;102(4):342-347.

37. Boujelbene N, Khabir A, Boujelbene N, Jeanneret Sozzi W, Mirimanoff RO, Khanfir K. Clinical review-breast adenoid cystic carcinoma. Breast. 2012;21(2):124-127.
38. Slodkowska E, Xu B, Kos Z, Bane A, Barnard M, Zubovits J, Iyengar $P$, et al. Predictors of outcome in mammary adenoid cystic carcinoma: a multi-institutional study. Am J Surg Pathol. 2020;44(2):214-223. 